\title{
3 Research Square

\section{Dual-Trigger Improves the Outcomes of in Vitro Fertilization Cycles in Older Patients with Diminished Ovarian Reserve: a retrospective cohort study}

\section{Chyi-Uei Chern}

Kaohsiung Veterans General Hospital

Ju-Yueh Li

Kaohsiung Veterans General Hospital

Kuan-Hao Tsui

Kaohsiung Veterans General Hospital

Peng-Hui Wang

Taipei Veterans General Hospital

Zhi-Hong Wen

National Sun Yat-sen University

Li-Te Lin ( $\sim$ litelin1982@gmail.com )

Kaohsiung Veterans General Hospital https://orcid.org/0000-0001-5063-6793

\section{Research}

Keywords: dual trigger, diminished ovarian reserve, in vitro fertilization, poor ovarian responders, POSEIDON group

Posted Date: March 11th, 2020

DOI: https://doi.org/10.21203/rs.3.rs-16738/v1

License: (c) (i) This work is licensed under a Creative Commons Attribution 4.0 International License. Read Full License

Version of Record: A version of this preprint was published at PLOS ONE on July 6th, 2020. See the published version at https://doi.org/10.1371/journal.pone.0235707. 


\section{Abstract}

Background: Dual-trigger for final oocyte maturation has been applied on the women with poor ovarian response or diminished ovarian reserve. However, the results were controversial. The Patient-Oriented Strategies Encompassing IndividualizeD Oocyte Number (POSEIDON) stratification is a set of newly established criteria for low prognosis patients. The aim of this study was to examine the effectiveness of dual trigger for final oocyte maturation on the in vitro fertilization (IVF) outcomes of patients who fulfill the POSEIDON group 4 criteria.

Methods: This retrospective cohort study investigated 384 cycles fulfilling the POSEIDON group 4 criteria. The patients underwent IVF treatment using the gonadotropin-releasing hormone ( $\mathrm{GnRH})$ antagonist protocol. The study group contained 194 cycles that received dual trigger (human chorionic gonadotropin [hCG] plus GnRH agonist) for final oocyte maturation. The control group included 114 cycles where final oocyte maturation was performed with only hCG. Baseline characteristics and cycle parameters, as well as IVF outcomes of both groups were compared.

Results: Baseline characteristics were similar between the dual trigger group and the control group. In terms of IVF outcomes, the dual trigger group demonstrated significantly higher number of retrieved oocytes, metaphase II oocytes, fertilized oocytes, day-3 embryos, and top-quality day-3 embryos. A statistically significant improvement in clinical pregnancy rate and live birth rate was also observed in the dual trigger group. Regardless of whether the patients were aged $<40$ or $\geq 40$ years, those receiving dual trigger had increased numbers of oocytes retrieved, fertilized oocytes, day-3 embryos, top-quality day-3 embryos than those with hCG-only trigger. Clinical pregnancy rate and live birth rate were significantly increased in the $<40$-year old group whilst not significantly increased in those $\geq 40$ years.

Conclusions: Our data suggests that dual trigger for final oocyte maturation might improve clinical pregnancy rates and live birth rates of IVF cycles in patients fulfilling the POSEIDON group 4 criteria.

\section{Introduction}

The process of in vitro fertilization (IVF) involves hyperstimulation of ovaries with gonadotropins to mimic the natural cycle in producing mature oocytes. In the normal menstrual cycle, oocyte maturation occurs following the luteinizing hormone (LH) and smaller follicle stimulating hormone (FSH) surge that happens during mid-cycle. Traditionally, human chorionic gonadotropin (hCG) has been the trigger of choice for oocyte maturation due to its molecular and biological similarity with LH [1]. However, its halflife is much longer than LH, lasting for approximately days [2]. This contributes to the occurrence of ovarian hyperstimulation syndrome (OHSS) in high responders. Also, hCG lacks FSH activity, which plays a role in the in vitro maturation of oocytes [3].

Gonadotropin releasing hormone $(\mathrm{GnRH})$ agonists were first suggested for final oocyte maturation by Gonen et al. in 1990, as it is able to trigger endogenous release of both FSH and LH [4]. With a shorter mean duration of LH surge of about 34 hours, it is similar to the natural cycle duration of 48 hours [5], 
effectively reducing the incidence of OHSS in high responders [6, 7]. However, some problems surfaced with the substitution of $\mathrm{GnRH}$ agonists as trigger. Kummer et al. [8] discovered that the risk of empty follicle syndrome was increased following isolated $\mathrm{GnRH}$ agonist trigger due to a suboptimal $\mathrm{LH}$ surge. Additionally, increased early pregnancy loss and decreased rates of ongoing pregnancy were noted by multiple studies [9, 10]. As such, the idea of a dual trigger was developed [11]. Indeed, the hCG component of dual trigger could serve as a rescue trigger in case of poor response to $\mathrm{GnRH}$ agonist, which occurs in about $2.71 \%$ of a study population [12].

Since its development, multiple investigations have shown the benefits of using a dual trigger for final oocyte maturation in normal responders [11,13], including an improvement in total number of retrieved oocytes, MII oocytes, rates of embryo implantation, clinical pregnancy, and live birth rates [14]. Evidence from available meta-analysis in 2018 involving four studies including 527 patients found a significantly improved clinical pregnancy rate following dual trigger [15]. However, for poor ovarian responders (PORs), the situation is less clear cut.

Women with poor ovarian response are those who have a reduced number of follicles responsive to FSH, resulting in poor IVF outcomes [16] - posing a grave challenge to clinicians worldwide. Numerous criteria have been proposed for the definition of POR [17], but none was established as the international standard to define POR until the creation of the Bologna criteria in 2011 [18]. Presence of much heterogeneity lead to strong criticism about the clinical application the Bologna criteria [19], triggering the development of the POSEIDON (Patient-Oriented Strategies Encompassing IndividualizeD Oocyte Number) criteria in 2016 [20]. The POSEIDON criteria brings forward the concept of "low prognosis", and classifies all patients according to 3 factors: Oocyte quality as demonstrated by age, oocyte quantity as represented by ovarian biomarkers of anti-Müllerian hormone (AMH) and/or antral follicle count (AFC), and finally ovarian response, dependent on the number of oocytes retrieved given a previous ovarian stimulation cycle. As such, it is able to aid the clinician in formulating a more tailored treatment plan for the individual groups. The focus of our study is POSEIDON group 4 , with patients who are $\geq 35$ years old, with AFC $<5$ and/or $\mathrm{AMH}<1.2 \mathrm{ng} / \mathrm{mL}$.

To our knowledge, there has not been a study that examines the benefit of dual trigger for patients who are classified under POSEIDON group 4. Therefore, we attempted to investigate the feasibility of utilizing dual trigger for final oocyte maturation in improving IVF outcomes of this population.

\section{Materials And Methods}

\section{Study Design and Participants}

The retrospective cohort study was performed at the reproductive medical center of Kaohsiung Veterans General Hospital, in Kaohsiung, Taiwan from January 2012 through December 2017. The study protocol was approved by the institutional review board at Kaohsiung Veterans General Hospital, with the identifier VGHKS19-CT8-05, and conforms to the "Declaration of Helsinki for Medical Research involving Human Subjects." Patients who underwent IVF cycles and fulfilled the criteria for POSEIDON group 4 (age $\geq 35$ 
years, with $\mathrm{AFC}<5$ and/or $\mathrm{AMH}<1.2 \mathrm{ng} / \mathrm{mL}$ ) were included in this study. A total of 384 cycles met the criteria during the study period. Exclusion criteria were as follows: (i) Patients who did not receive GnRH antagonist protocol, (ii) patients with premature ovarian insufficiency, (iii) patients over the age of 45, (iv) cancer patients who have received chemotherapy or radiotherapy, and (v) patients with incomplete data. Following application of the exclusion criteria, a total of 308 cycles were included for study and then divided into dual-trigger and hCG trigger groups. The choice of hCG alone or dual trigger depended on the physician's preference. In the dual-trigger group $(n=194)$, patients received final oocyte maturation with GnRH agonist and hCG. In the hCG trigger group $(n=114)$, patients received only hCG for final oocyte maturation. The study flow chart is shown in Figure 1.

\section{Treatment Protocol}

Only patients receiving GnRH antagonist protocol were included in this study. Following a baseline hormone screen and transvaginal ultrasound for antral follicles, controlled ovarian stimulation was initiated within 5 days of the menstrual cycle, with recombinant follicle stimulating hormone ( $\mathrm{rFSH}$, Gonal-F, Merck Serono S.p.A., Modugno, Italy), rFSH plus recombinant luteinizing hormone (Pergoveris, Merck Serono SA, Aubonne, Switzerland), human menopausal gonadotropin (Merional, IBSA Institut Biochimique S.A., Lamone, Switzerland), or corifollitropin alfa (Elonva, Vetter Pharma-Fertigung GmbH \& Co, KG, Ravensburg, Germany).

Patient response was monitored during the IVF cycle with serial transvaginal ultrasound for follicular measurements and hormone profiles. Dosage was adjusted depending on follicular response and previous response to gonadotropins. Daily GnRH antagonist injections (Cetrotide $0.25 \mathrm{mg}$, Pierre Fabre Medicament Production, Aquitaine Pharm International, Idron, France or Orgalutran $0.25 \mathrm{mg}$, Vetter Pharma-Fertigung GmbH \& Co, KG, Ravensburg, Germany) were administered when the leading follicle reaches 12-14 $\mathrm{mm}$ in diameter, up till the date of final oocyte maturation. Patient then either received dual trigger with combined recombinant hCG (Ovidrel 250 $\mu$ g, Merck Serono S.p.A., Modugno, Italy) with GnRH agonist (Lupro 2mg, Nang Kuang Pharmaceutical Co, Ltd., Tainan, Taiwan) or recombinant hCG alone. Trigger is given when at least one leading follicle reaches a mean diameter of $18 \mathrm{~mm}$.

Transvaginal ultrasound guided oocyte retrieval was performed $36 \mathrm{~h}$ following administration of trigger. Whether fertilization was conducted by conventional IVF or intracytoplasmic sperm injection depended on semen analysis results or prior fertilization condition. Embryos were evaluated and graded according to the criteria established by the Istanbul consensus workshop [21]. All embryos were cryopreserved by vitrification on the third day after oocyte retrieval for subsequent frozen embryo transfer. An artificial cycle was used for endometrial preparation. Endometrium was prepared with daily oral estradiol (Ediol 8mg, Synmosa Biopharma Corporation, Hsinchu County, Taiwan) and estradiol gel (Oestrogel gel, Besins, Drogenbos, Belgium) beginning before cycle day 5 .

When the endometrial thickness reaches at least $8 \mathrm{~mm}$, daily progesterone, including intravaginal gel (Crinone 8\% gel, Merck Serono, Hertfordshire, UK) and oral dydrogesterone (Duphaston 40mg, Abbott, Olst, the Netherlands), were given simultaneously. Then, cryopreserved embryo transfer was done under 
transabdominal sonographic assistance. Progesterone supplementation was administered until 8-10 gestational weeks upon confirmation of pregnancy.

\section{Outcome Measures}

The primary outcome measure was live birth rate, defined as the delivery of a viable fetus beyond 24 weeks of gestation. Secondary outcome measures included the number of retrieved oocytes, number of mature oocytes, number of fertilized oocytes, number of day-3 embryos, number of top- quality day-3 embryos, and clinical pregnancy rate. Clinical pregnancy rate was defined by the presence of a fetal heartbeat at 6-7 weeks of a pregnancy.

\section{Statistical Analysis}

Kolmogovor-Smirnov test was used to evaluate normality of quantitative variables. Quantitative variables were evaluated using the independent t-test. Chi-Square test was used to evaluate categorical variables. Odds ratios (ORs) and 95\% confidence intervals (Cls) of clinical pregnancy and live birth were assessed using binary logistic regression, after adjusting for confounders. Analyses were performed using the Statistical Package for Social Sciences (SPSS) version 20.0 (Chicago, IL, USA). $P<0.05$ were considered statistically significant.

\section{Results}

As shown in Figure 1, out of 2,165 IVF cycles, 384 cycles fulfilled the POSEIDON group 4 criteria. Of the 384 cycles, 38 were not treated using a GnRH antagonist protocol, 8 were diagnosed with premature ovarian insufficiency, 19 with advanced maternal age of more than 45 years old, 3 were cancer patients who underwent chemotherapy and/or radiotherapy, and 8 had incomplete dataset. Those cycles were excluded from the study.

A total of 308 cycles were included in the study, with 194 belonging to the dual-trigger group and 114 in the hCG-only trigger group. Comparisons between the populations of two groups revealed no difference in patient age, body mass index, infertility duration, previous IVF attempts, primary or secondary infertility, basal FSH, AFC and AMH (Table 1).

Cycle characteristics and pregnancy outcome between the two groups are presented in Table 2. There were no differences in terms of stimulation duration or total gonadotropin dose, irrespective of corifollitropin alfa use. The number of retrieved oocytes ( $3.3 \pm 2.7$ vs. $1.6 \pm 1.9, p<0.001)$, metaphase II oocytes ( $2.5 \pm 1.9$ vs. $1.2 \pm 1.4, p<0.001)$, fertilized oocytes ( $2.3 \pm 2.1$ vs. $1.1 \pm 1.4, p<0.001)$, day-3 embryos $(2.2 \pm 2.0$ vs. $1.0 \pm 1.3, p<0.001)$ and top-quality day-3 embryos $(0.9 \pm 1.3$ vs. $0.2 \pm 0.5, p<0.001)$ were significantly higher in the dual trigger group compared with the hCG-only group. Of note, the dual-trigger group performed superiorly in terms of both clinical pregnancy rate $(19.1 \%$ vs. $7.0 \%, p=0.004)$ and live birth rate $(14.4 \%$ vs. $4.4 \%, p=0.006)$. Fertilization rate was similar between the two groups. 
Binary logistic regression was performed to determine whether dual-trigger use had a beneficial effect on clinical pregnancy rate and live birth rate in Table 3 and Table 4, respectively. Confounding variables such as age, body mass index, duration of infertility, previous IVF attempts, types of infertility, basal FSH, AFC and $\mathrm{AMH}$ were included in the analysis. The multivariate analysis showed that dual trigger use $(\mathrm{OR}=$ $3.58,95 \% \mathrm{Cl} 1.26-10.17, p=0.017)$ and $\mathrm{AMH}(\mathrm{OR}=5.62,95 \% \mathrm{Cl} 1.16-27.17, p=0.032)$ were positively associated with clinical pregnancy rate (Table 3$)$. However, only dual trigger use $(\mathrm{OR}=4.18,95 \% \mathrm{Cl} 1.11$ $15.83, p=0.035$ ) was a positively independent factor for live birth rate.

As shown in Table 5, regardless of whether the patients were aged $<40$ or $\geq 40$ years, those receiving dual-trigger had increased numbers of oocytes retrieved, fertilized oocytes, day-3 embryos, top-quality day-3 embryos than those with hCG-only trigger. Clinical pregnancy rate $(22.8 \%$ vs. $5.8 \%, p=0.009)$ and live birth rate $(19.0 \%$ vs. $3.8 \%, p=0.012)$ were significantly increased in the $<40$-year old group whilst not significantly in the $\geq 40$-year old group.

\section{Discussion}

This retrospective cohort study is the first to assess the effects of dual-trigger on IVF outcomes in patients fulfilling the POSEIDON group 4 criteria. Our study showed that dual-trigger is superior to hCG administration alone for final oocyte maturation in producing increased numbers of retrieved oocytes, metaphase II oocytes, fertilized oocytes, day-3 embryos and top-quality day-3 embryos. Clinical pregnancy rate and live birth rate were also improved with dual-trigger administration. Moreover, the multivariate analysis revealed a 3.58-fold increase in clinical pregnancy $(95 \% \mathrm{Cl} 1.26-10.17, p=0.017)$ and a 4.18-fold increase in live birth $(95 \% \mathrm{Cl} 1.11-15.83, p=0.035)$ in the POSEIDON group 4 patients with dual trigger compared to those with hCG trigger. When the population is further divided into subgroups of $<40$ and $\geq 40$ year olds, we found a significant improvement across all abovementioned parameters for the patients $<40$ years. For the subpopulation $\geq 40$ years, a significant increase was seen in number of oocytes retrieved, fertilized oocytes, number of day-3 embryos and top-quality day-3 embryos. Clinical pregnancy rate and live birth rate for this population also trended towards improvement, although not statistically significant.

Similar outcomes have been obtained in the study of patients with diminished ovarian reserve or poor ovarian reserve in recent years, showing a beneficial effect of dual-trigger in improving IVF outcomes. Lin et al. [22] confirmed in a recent retrospective cohort study involving $427 \mathrm{GnRH}$ antagonist IVF cycles with fresh embryo transfer that dual-triggering significantly increases the live birth rate $(26.9 \%$ vs $14.5 \%, p=$ $0.014)$, clinical pregnancy rate $(33.0 \%$ vs. $20.7 \%, p=0.035)$, and fertilization rate $(73.1 \%$ vs. $58.6 \%, p=$ $0.015)$ in women with diminished ovarian reserve, compared to hCG-alone trigger. In an even larger retrospective cohort study with 1389 IVF cycles fulfilling the Bologna criteria, utilizing the progesteroneprimed ovarian stimulation protocol, Zhang et al. [23] also reports significantly higher number of oocytes collected $(p<0.001)$ with improved number of mature oocytes $(p<0.001)$. However, other studies have found dual-trigger to be ineffective at improving IVF outcomes for this population. Eser et al. [24], in a case control study involving 47 dual-trigger and 62 hCG only trigger cases fulfilling the Bologna criteria 
for POR, discovered no statistical different between the two groups with reference to implantation rate, biochemical pregnancy rate, clinical pregnancy rate, and ongoing pregnancy rate. Due to the divergence of opinion on the effectiveness and clinical utility of dual-trigger for final oocyte maturation, large scale randomized controlled trials are required to reach a verdict.

In combining GnRH agonist and hCG for the final oocyte trigger, we are in essence enjoying the best of both worlds. Despite being molecularly similar, hCG and LH elicit different gene expressions. LH tends towards cellular growth, which supports embryo development and survival, whereas hCG enhances apoptosis $[25,26]$. hCG administration alone also does not produce FSH activity, while GnRH agonist releases an endogenous FSH and LH surge, resulting in a more physiologic response. In vitro studies have highlighted the role of FSH in oocyte maturation [3], via the increased production of epiregulin (Ereg) and amphiregulin (Areg) [27], members of the epidermal growth factor-like (EGF) family, which have been shown to mediate the LH signal and participate in cumulus expansion and oocyte maturation [28]. This has been confirmed in vitro experiments, where Ereg and Areg presence in maturation medium helps to improve the maturation rate of human GV oocytes [29]. Animal studies have also shown that FSH has the independent ability of inducing ovulation [30], perhaps by stimulating plasminogen activator activity, which converts plasminogen into active protease plasmin, helping to weaken the follicular wall and aiding rupture and oocyte dissociation $[3,31,32]$. Also, the $\mathrm{FSH}$ surge induces $\mathrm{LH}$ formation on luteinized granulosa cells, promoting oocyte maturation and cumulus expansion [33]. Clinically, randomized controlled trials have shown that administration of FSH along with hCG trigger increases the rate of oocyte recovery and improved the fertilization rate (40). In their study, 188 women undergoing a long agonist suppression protocol for treatment of infertility were randomized to receive a FSH bolus versus placebo at time of hCG trigger. Fertilization rates were significantly higher (63\% vs. $55 \%)$ in the treatment arm, with increased likelihood of oocyte recovery ( $70 \%$ vs. $57 \%)$. However, this did not translate into a difference in clinical pregnancy rate or live birth rate.

Additionally, direct $\mathrm{GnRH}$ receptor activation as identified by Raga et al. could also have an effect on preimplantation embryonic development that is unrelated to FSH activity [34]. The GnRH receptor expression was found to be greatest in granulosa cells [35], and in rat models, administration of GnRH agonists induced an increase in receptor levels in a dose-dependent manner, whereas LH decreased GnRH receptor mRNA levels [36]. Administration of GnRH agonist trigger has also demonstrated the retrieval of more MIl oocytes (16\%) [9]. As such, the triggering cocktail of hCG, FSH, LH and GnRH agonist serves to provide the benefits of a multi-faceted approach.

In our study, the patients who stand to benefit the most from such a trigger regimen appear to be those less than 40-years of age. Other possible subgroups include those with low proportion of mature oocytes $(<66 \%)$ per number oocytes retrieved [37], where it was demonstrated that patients who received dualtrigger had significantly higher number of Mll oocytes (6.5 vs. 3.6, $p<0.008)$, number of oocytes retrieved $(69.7 \%$ vs. $47.1 \%, p<0.03)$, and a higher number of top-quality embryos (3.1 vs. $1, p<0.02)$ [38]. Moreover, those with history of poor fertilization, as defined by fertilization rate $<20 \%$ in at least two prior ICSI cycle, are also a potential benefit of such a combination. In their retrospective cohort study, Elias et 
al. [39] found in 2017 that the mean fertilization rate in the combined trigger group was found to be significantly higher $16.4 \%$ (95\% Cl: $7.58 \%-25.2 \%)$, with higher oocyte maturity ( $82.1 \%$ vs. $69.8 \%)$, higher clinical pregnancy $(27.5 \%$ vs. $5.67 \%)$, and higher live birth rates $(20.2 \%$ vs. $3.46 \%)$ compared to the hCG trigger group.

The relatively small sample size of this study along with its retrospective design poses major limitations. We are underpowered to analyse if dual-trigger has an impact on IVF outcomes of patients aged less than or more than 40 years old. Another shortcoming of our study is that despite all cycles being antagonist cycles, we only took into account total gonadotropin dosage, and there is considerable heterogeneity in exact type of gonadotropin used. The strength of our study is that all clinical decisions and oocyte pickups were performed by the same physician, leading to less variability in performance.

In conclusion, our data suggest that dual trigger might increase both oocyte and embryo yields, as well as clinical pregnancy rates and live birth rates in patients fulfilling the POSEIDON group 4 criteria. However, large-scale randomized controlled studies are needed to confirm these findings.

\section{Abbreviations}

AFC: antral follicle count; AMH: anti-Müllerian hormone; Cl: confidence interval; FSH: follicle stimulation hormone; GnRH: gonadotropin-releasing hormone; hCG: human chorionic gonadotropin; IVF: in vitro fertilization; LH: luteinizing hormone; OHSS: ovarian hyperstimulation syndrome; OR: odd ratio; POR: poor ovarian responder; POSEIDON: Patient-Oriented Strategies Encompassing IndividualizeD Oocyte Number

\section{Declarations}

\section{Ethics approval and consent to participate}

The study conformed to the "Declaration of Helsinki for Medical Research involving Human Subjects". Additionally, approval was obtained from the institutional review board at Kaohsiung Veterans General Hospital, with the identifier VGHKS19-CT8-05. The study was performed in accordance with approved guidelines.

\section{Consent for publication}

Not applicable.

\section{Availability of data and material}

The datasets used and analyzed during the current study are available from the corresponding author on reasonable request.

\section{Competing interests}


The authors declare that the research was conducted in the absence of any commercial or financial relationships that could be construed as a potential conflict of interest.

\section{Funding}

No funding.

\section{Authors' contributions}

$\mathrm{PH}$ and $\mathrm{ZH}$ contributed the conception and design of the study; JY and CU organized the database; LT performed the statistical analysis; CU wrote the first draft of the manuscript; LT and KH wrote sections of the manuscript. All authors contributed to manuscript revision, read and approved the submitted version.

\section{Acknowledgements}

This work was generously supported by grants VGHKS19-CT8-05 from Kaohsiung Veterans General Hospital.

\section{References}

1. Ascoli M, Fanelli F, Segaloff DL. The lutropin/choriogonadotropin receptor, a 2002 perspective. Endocr Rev. 2002;23(2):141-174.

2. Gonen Y, Balakier, H., Powell, W., Casper, R.F. Use of gonadotropin-releasing hormone agonist to trigger follicular maturation for in vitro fertilization. J Clin Endocrinol Metab. 1990;71(4):918-922.

3. Strickland S, Beers WH. Studies on the role of plasminogen activator in ovulation. In vitro response of granulosa cells to gonadotropins, cyclic nucleotides, and prostaglandins. J Biol Chem. 1976;251(18):5694-5702.

4. Humaidan $P$, Bungum $M$, Bungum $L$, Yding Andersen $C$. Effects of recombinant $L H$ supplementation in women undergoing assisted reproduction with $\mathrm{GnRH}$ agonist down-regulation and stimulation with recombinant FSH: an opening study. Reprod Biomed Online. 2004;8(6):635-643.

5. Hoff JD. QM, Yen SS. Hormonal dynamics at midcycle: a reevaluation. J Clin Endocrinol Metab. 1983;57(4):792-796.

6. Engmann L, DiLuigi, A., Schmidt, D., . The use of gonadotropin-releasing hormone (GnRH) agonist to induce oocyte maturation after cotreatment with $\mathrm{GnRH}$ antagonist in high-risk patients undergoing in vitro fertilization prevents the risk of ovarian hyperstimulation syndrome: a prospective randomized controlled study. Fertil Steril. 2008;89(1):84-91.

7. Griesinger G. vOS, Schroer A., Ludwig AK., Diedrich K., Al-Hasani S., Schultze-Mosgau A.,. Elective cryopreservation of all pronuclear oocytes after $\mathrm{GnRH}$ agonist triggering of final oocyte maturation in patients at risk of developing OHSS. Hum Reprod. 2007;22(5):1348-1352.

8. Kummer NE, Feinn RS, Griffin DW, Nulsen JC, Benadiva CA, Engmann LL. Predicting successful induction of oocyte maturation after gonadotropin-releasing hormone agonist ( $\mathrm{GnRHa}$ ) trigger. Hum 
Reprod. 2013;28(1):152-159.

9. Humaidan P, Bredkjaer HE, Bungum L, et al. GnRH agonist (buserelin) or hCG for ovulation induction in GnRH antagonist IVF/ICSI cycles: a prospective randomized study. Hum Reprod. 2005;20(5):12131220.

10. Kolibianakis EM, Schultze-Mosgau A, Schroer A, et al. A lower ongoing pregnancy rate can be expected when $\mathrm{GnRH}$ agonist is used for triggering final oocyte maturation instead of HCG in patients undergoing IVF with GnRH antagonists. Hum Reprod. 2005;20(10):2887-2892.

11. Shapiro BS, Daneshmand ST, Garner FC, Aguirre M, Thomas S. Gonadotropin-releasing hormone agonist combined with a reduced dose of human chorionic gonadotropin for final oocyte maturation in fresh autologous cycles of in vitro fertilization. Fertil Steril. 2008;90(1):231-233.

12. Lu X, Hong Q, Sun $L$, et al. Dual trigger for final oocyte maturation improves the oocyte retrieval rate of suboptimal responders to gonadotropin-releasing hormone agonist. Fertil Steril. 2016;106(6):13561362.

13. Shapiro BS, Daneshmand ST, Garner FC, Aguirre M, Hudson C. Comparison of "triggers" using leuprolide acetate alone or in combination with low-dose human chorionic gonadotropin. Fertil Steril. 2011;95(8):2715-2717.

14. Lin MH, Wu FS, Lee RK, Li SH, Lin SY, Hwu YM. Dual trigger with combination of gonadotropinreleasing hormone agonist and human chorionic gonadotropin significantly improves the live-birth rate for normal responders in GnRH-antagonist cycles. Fertil Steril. 2013;100(5):1296-1302.

15. Chen $\mathrm{CH}$, Tzeng $\mathrm{CR}$, Wang $\mathrm{PH}$, et al. Dual triggering with $\mathrm{GnRH}$ agonist plus hCG versus triggering with hCG alone for IVF/ICSI outcome in GnRH antagonist cycles: a systematic review and metaanalysis. Arch Gynecol Obstet. 2018;298(1):17-26.

16. Esteves SC, Roque M, Bedoschi GM, Conforti A, Humaidan P, Alviggi C. Defining Low Prognosis Patients Undergoing Assisted Reproductive Technology: POSEIDON Criteria-The Why. Front Endocrinol (Lausanne). 2018;9:461.

17. Polyzos NP, Devroey P. A systematic review of randomized trials for the treatment of poor ovarian responders: is there any light at the end of the tunnel? Fertil Steril. 2011;96(5):1058-1061 e1057.

18. Ferraretti AP, La Marca A, Fauser BC, et al. ESHRE consensus on the definition of 'poor response' to ovarian stimulation for in vitro fertilization: the Bologna criteria. Hum Reprod. 2011;26(7):1616-1624.

19. Younis JS, Ben-Ami M, Ben-Shlomo I. The Bologna criteria for poor ovarian response: a contemporary critical appraisal. J Ovarian Res. 2015;8:76.

20. Humaidan P, Alviggi C, Fischer R, Esteves SC. The novel POSEIDON stratification of 'Low prognosis patients in Assisted Reproductive Technology' and its proposed marker of successful outcome. F1000Res. 2016;5:2911.

21. Medicine ASiR, Embryology ESIGo. The Istanbul consensus workshop on embryo assessment: proceedings of an expert meetingt. Human Reproduction. 2011;26(6):1270-1283.

22. Chen SN, Wang PH, Hsieh MF, Tsai HW, Lin LT, Tsui KH. Maternal pregnancy-induced hypertension increases the subsequent risk of neonatal candidiasis: A nationwide population-based cohort study. 
Taiwan J Obstet Gynecol. 2019;58(2):261-265.

23. Zhang J, Wang $Y$, Mao $X$, et al. Dual trigger of final oocyte maturation in poor ovarian responders undergoing IVF/ICSI cycles. Reprod Biomed Online. 2017;35(6):701-707.

24. Eser A, Devranoglu B, Bostanci Ergen E, Yayla Abide C. Dual trigger with gonadotropin-releasing hormone and human chorionic gonadotropin for poor responders. J Turk Ger Gynecol Assoc. 2018;19(2):98-103.

25. Grondahl ML, Borup R, Lee YB, Myrhoj V, Meinertz H, Sorensen S. Differences in gene expression of granulosa cells from women undergoing controlled ovarian hyperstimulation with either recombinant follicle-stimulating hormone or highly purified human menopausal gonadotropin. Fertil Steril. 2009;91(5):1820-1830.

26. Ruvolo G, Bosco L, Pane A, Morici G, Cittadini E, Roccheri MC. Lower apoptosis rate in human cumulus cells after administration of recombinant luteinizing hormone to women undergoing ovarian stimulation for in vitro fertilization procedures. Fertil Steril. 2007;87(3):542-546.

27. Haas J, Ophir L, Barzilay E, et al. Standard human chorionic gonadotropin versus double trigger for final oocyte maturation results in different granulosa cells gene expressions: a pilot study. Fertil Steril. 2016;106(3):653-659 e651.

28. Park J, Su Y, Ariga M, Law E, Jin S, Conti M. EGF-like growth factors as mediators of LH action in the ovulatory follicle. Science. 2004;303:682-684.

29. Ben-Ami I, Komsky A, Bern O, Kasterstein E, Komarovsky D, Ron-El R. In vitro maturation of human germinal vesicle-stage oocytes: role of epidermal growth factor-like growth factors in the culture medium. Hum Reprod. 2011;26(1):76-81.

30. Zelinksi-Wooten MB, Hutchison, J.S., Wolf, D.P., Stouffer, R.L. A bolus of recombinant human follicle stimulating hormone at midcycle induces periovulatory events following multiple follicular development in macaques. Hum Reprod. 1998;13(3):554-560.

31. Morioka N, Zhu C, Brannstrom M, Woessner JF, LeMaire WJ. Mechanism of mammalian ovulation. Prog Clin Biol Res. 1989;294:65-85.

32. Lamb JD, Shen S, McCulloch C, Jalalian L, Cedars MI, Rosen MP. Follicle-stimulating hormone administered at the time of human chorionic gonadotropin trigger improves oocyte developmental competence in in vitro fertilization cycles: a randomized, double-blind, placebo-controlled trial. Fertil Steril. 2011;95(5):1655-1660.

33. Humaidan P, Kol S, Papanikolaou EG, Copenhagen Gn RHATWG. GnRH agonist for triggering of final oocyte maturation: time for a change of practice? Hum Reprod Update. 2011;17(4):510-524.

34. Raga F, Bonilla-Musoles F, Casan EM, Bonilla F. Recombinant follicle stimulating hormone stimulation in poor responders with normal basal concentrations of follicle stimulating hormone and oestradiol: improved reproductive outcome. Hum Reprod. 1999;14(6):1431-1434.

35. Bauer-Dantoin AC, Weiss J, Jameson JL. Roles of estrogen, progesterone, and gonadotropinreleasing hormone $(\mathrm{GnRH})$ in the control of pituitary $\mathrm{GnRH}$ receptor gene expression at the time of the preovulatory gonadotropin surges. Endocrinology. 1995;136(3):1014-1019. 
36. Limonta P, Moretti RM, Montagnani Marelli M, Motta M. The biology of gonadotropin hormonereleasing hormone: role in the control of tumor growth and progression in humans. Front Neuroendocrinol. 2003;24(4):279-295.

37. Griffin D, Feinn R, Engmann L, Nulsen J, Budinetz T, Benadiva C. Dual trigger with gonadotropinreleasing hormone agonist and standard dose human chorionic gonadotropin to improve oocyte maturity rates. Fertil Steril. 2014;102(2):405-409.

38. Zilberberg E, Haas J, Dar S, Kedem A, Machtinger R, Orvieto R. Co-administration of GnRH-agonist and $\mathrm{hCG}$, for final oocyte maturation (double trigger), in patients with low proportion of mature oocytes. Gynecol Endocrinol. 2015;31(2):145-147.

39. Elias RT, Pereira N, Artusa L, et al. Combined GnRH-agonist and human chorionic gonadotropin trigger improves ICSI cycle outcomes in patients with history of poor fertilization. J Assist Reprod Genet. 2017;34(6):781-788.

\section{Tables}

Table 1 Baseline characteristics of the older patients with diminished ovarian reserve (POSEIDIN group 4) with dual-trigger or hCG trigger

\begin{tabular}{llll}
\hline Parameters & $\begin{array}{l}\text { Dual trigger } \\
(\mathrm{n}=194)\end{array}$ & $\begin{array}{l}\text { hCG trigger } \\
(\mathrm{n}=114)\end{array}$ & $p$ value \\
& & \\
\hline Age (years) & $40.0 \pm 2.7$ & $40.0 \pm 2.8$ & 0.948 \\
Body mass index (kg/m2) & $22.9 \pm 3.5$ & $22.4 \pm 2.9$ & 0.170 \\
Infertility duration (year) & $5.6 \pm 4.6$ & $5.1 \pm 4.3$ & 0.402 \\
Previous IVF attempts (n) & $2.2 \pm 2.4$ & $1.8 \pm 2.1$ & 0.195 \\
Types of infertility (\%) & & & 0.335 \\
$\quad$ Primary infertility & 55.7 & 50 & \\
$\quad$ Secondary infertility & 44.3 & 50 & \\
Basal FSH (IU/l) & $6.3 \pm 3.8$ & $7.0 \pm 3.3$ & 0.158 \\
Antral follicle counts (n) & $2.4 \pm 1.3$ & $2.5 \pm 1.7$ & 0.425 \\
Anti-Müllerian hormone (ng/ml) & $0.48 \pm 0.26$ & $0.47 \pm 0.31$ & 0.857 \\
\hline
\end{tabular}

Data are presented as mean \pm standard deviation and \%.

IVF, in vitro fertilization ; FSH, follicle stimulation hormone

Table 2 Cycle characteristics and pregnancy outcome of the older patients with diminished ovarian reserve (POSEIDIN group 4) with dual-trigger or hCG trigger 
Parameters

Dual trigger hCG trigger $\quad p$ value

$(\mathrm{n}=194) \quad(\mathrm{n}=114)$

\begin{tabular}{llll}
\hline $\begin{array}{l}\text { Stimulation duration (days) } \\
\text { Gonadotropin dosage (IU) }\end{array}$ & $10.6 \pm 2.2$ & $10.4 \pm 2.7$ & 0.445 \\
$\quad$ with corifollitropin alfa & $1751.4 \pm 708.9$ & $1561.4 \pm 676.6$ & 0.316 \\
without corifollitropin alfa & $2931.5 \pm 779.9$ & $2729.7 \pm 1109.0$ & 0.129 \\
No. of oocytes retrieved (n) & $3.3 \pm 2.7$ & $1.6 \pm 1.9$ & $<0.001$ \\
No. of metaphase II oocytes (n) & $2.5 \pm 1.9$ & $1.2 \pm 1.4$ & $<0.001$ \\
No. of fertilized oocytes (n) & $2.3 \pm 2.1$ & $1.1 \pm 1.4$ & $<0.001$ \\
Fertilization rate (\%) & $72.7 \pm 29.0$ & $73.1 \pm 35.3$ & 0.934 \\
No. of Day 3 embryos (n) & $2.2 \pm 2.0$ & $1.0 \pm 1.3$ & $<0.001$ \\
No. of top-quality Day 3 embryos (n) & $0.9 \pm 1.3$ & $0.2 \pm 0.5$ & $<0.001$ \\
Clinical pregnancy rate (\%) & 19.1 & 7.0 & 0.004 \\
Live birth rate (\%) & 14.4 & 4.4 & 0.006 \\
\hline
\end{tabular}

Data are presented as mean \pm standard deviation and \%.

Table 3 Analyses of factors affecting clinical pregnancy rate in in older patients with diminished ovarian reserve (POSEIDIN group 4)

\begin{tabular}{lcclc}
\hline & \multicolumn{2}{c}{ Univariate analysis } & \multicolumn{2}{c}{ Multivariate analysis } \\
\cline { 2 - 5 } & OR (95\% CI) & $p$ value & $\begin{array}{c}\text { Adjusted OR } \\
\text { (95\% CI) }\end{array}$ & $p$ value \\
\hline Dual vs. hCG trigger & $3.12(1.40-6.97)$ & 0.005 & $3.58(1.26-10.17)$ & 0.017 \\
Age, years & $0.91(0.81-1.02)$ & 0.107 & $0.93(0.80-1.08)$ & 0.364 \\
\hline BMI, kg/m2 & $1.07(0.97-1.18)$ & 0.154 & $1.03(0.92-1.16)$ & 0.567 \\
\hline Infertility duration & $0.93(0.85-1.01)$ & 0.080 & $0.97(0.88-1.06)$ & 0.472 \\
\hline Previous IVF attempts, n $0.99(0.86-1.13)$ & 0.830 & $0.98(0.82-1.17)$ & 0.820 \\
\hline Types of infertility & & & & \\
\hline \multicolumn{1}{c}{ Primary vs. Secondary $0.99(0.53-1.86)$} & 0.972 & $1.12(0.50-2.51)$ & 0.781 \\
\hline Basal FSH, IU/l & $1.05(0.96-1.14)$ & 0.285 & $0.98(0.86-1.11)$ & 0.764 \\
\hline AFC, n & $1.19(0.97-1.46)$ & 0.102 & $1.01(0.75-1.34)$ & 0.967 \\
\hline AMH, ng/ml & $4.34(1.25-15.14)$ & 0.021 & $5.62(1.16-27.17)$ & 0.032 \\
\hline
\end{tabular}

$\mathrm{OR}$, odd ratio; $\mathrm{Cl}$, confidence interval; $\mathrm{BMI}$, body mass index; IVF, in vitro fertilization; FSH, follicle stimulation hormone; $\mathrm{AFC}$, antral follicle counts; $\mathrm{AMH}$, anti-Müllerian hormone. 
Table 4 Analyses of factors affecting live birth rate in in older patients with diminished ovarian reserve (POSEIDIN group 4)

\begin{tabular}{lcccc}
\hline & \multicolumn{2}{c}{ Univariate analysis } & \multicolumn{2}{c}{ Multivariate analysis } \\
\cline { 2 - 5 } & OR (95\% CI) & $p$ value & $\begin{array}{c}\text { Adjusted OR } \\
\text { (95\% CI) }\end{array}$ & $p$ value \\
\hline Dual vs. hCG trigger & $3.68(1.38-9.82)$ & 0.009 & $4.18(1.11-15.83)$ & 0.035 \\
Age, years & $0.87(0.76-0.99)$ & 0.041 & $0.88(0.74-1.05)$ & 0.170 \\
\hline BMI, kg/m2 & $1.09(0.98-1.22)$ & 0.102 & $1.04(0.91-1.18)$ & 0.556 \\
\hline Infertility duration & $0.91(0.82-1.02)$ & 0.093 & $0.97(0.86-1.09)$ & 0.614 \\
\hline Previous IVF attempts, n $0.90(0.75-1.08)$ & 0.246 & $0.88(0.68-1.14)$ & 0.321 \\
\hline Types of infertility & & & & \\
\hline \multicolumn{1}{c}{ Primary vs. Secondary $1.05(0.51-2.16)$} & 0.905 & $1.59(0.59-4.29)$ & 0.362 \\
\hline Basal FSH, IU/l & $1.06(0.96-1.16)$ & 0.246 & $0.94(0.80-1.09)$ & 0.390 \\
\hline AFC, n & $1.26(1.01-1.58)$ & 0.042 & $1.11(0.80-1.55)$ & 0.522 \\
\hline AMH, ng/ml & $3.99(0.95-16.78)$ & 0.059 & $5.58(0.83-37.71)$ & 0.078 \\
\hline
\end{tabular}

$\mathrm{OR}$, odd ratio; $\mathrm{Cl}$, confidence interval; $\mathrm{BMI}$, body mass index; IVF, in vitro fertilization; $\mathrm{FSH}$, follicle stimulation hormone; AFC, antral follicle counts; $\mathrm{AMH}$, anti-Müllerian hormone.

Table 5 Subgroup analyses (categorized relative to the age of 40 ) of older patients with diminished ovarian reserve (POSEIDIN group 4) with dual-trigger or hCG trigger 


\begin{tabular}{|c|c|c|c|c|c|c|}
\hline \multirow[b]{2}{*}{ Parameters } & \multicolumn{3}{|c|}{$<40(35 \sim 39)$ y/o } & \multicolumn{3}{|c|}{$40(40 \sim 45)$ y/o } \\
\hline & $\begin{array}{l}\text { Dual trigger } \\
\qquad(\mathrm{n}=79)\end{array}$ & $\begin{array}{l}\text { hCG trigger } \\
\qquad(\mathrm{n}=52)\end{array}$ & $p$ value & $\begin{array}{l}\text { Dual trigger } \\
\qquad(\mathrm{n}=115)\end{array}$ & $\begin{array}{l}\text { hCG trigger } \\
\qquad(\mathrm{n}=62)\end{array}$ & $p$ value \\
\hline Age (years) & $37.3 \pm 1.5$ & $37.5 \pm 1.3$ & 0.573 & $41.8 \pm 1.6$ & $42.1 \pm 1.7$ & 0.340 \\
\hline $\begin{array}{l}\text { Body mass } \\
\text { index }(\mathrm{kg} / \mathrm{m} 2)\end{array}$ & $22.4 \pm 3.7$ & $21.9 \pm 3.0$ & 0.368 & $23.2 \pm 3.3$ & $22.8 \pm 2.7$ & 0.389 \\
\hline $\begin{array}{l}\text { Basal FSH } \\
(\mathrm{IU} / \mathrm{l})\end{array}$ & $6.1 \pm 3.6$ & $7.5 \pm 3.8$ & 0.074 & $6.4 \pm 3.9$ & $6.6 \pm 2.9$ & 0.730 \\
\hline $\begin{array}{l}\text { Antral follicle } \\
\text { counts (n) }\end{array}$ & $2.6 \pm 1.3$ & $2.9 \pm 1.8$ & 0.426 & $2.2 \pm 1.2$ & $2.3 \pm 1.6$ & 0.865 \\
\hline $\begin{array}{l}\text { Anti-Müllerian } \\
\text { hormone } \\
\text { (ng/ml) }\end{array}$ & $0.48 \pm 0.24$ & $0.51 \pm 0.31$ & 0.591 & $0.48 \pm 0.27$ & $0.45 \pm 0.32$ & 0.464 \\
\hline $\begin{array}{l}\text { No. of oocytes } \\
\text { retrieved (n) }\end{array}$ & $4.1 \pm 2.7$ & $1.5 \pm 1.7$ & $\begin{array}{c}< \\
0.001\end{array}$ & $2.7 \pm 2.5$ & $1.7 \pm 2.1$ & 0.011 \\
\hline $\begin{array}{l}\text { No. of } \\
\text { metaphase II } \\
\text { oocytes (n) }\end{array}$ & $3.2 \pm 1.8$ & $1.2 \pm 1.2$ & $\begin{array}{c}< \\
0.001\end{array}$ & $2.1 \pm 1.8$ & $1.2 \pm 1.5$ & 0.001 \\
\hline $\begin{array}{l}\text { No. of } \\
\text { fertilized } \\
\text { oocytes }(n)\end{array}$ & $2.9 \pm 2.3$ & $1.0 \pm 1.1$ & $\begin{array}{c}< \\
0.001\end{array}$ & $1.9 \pm 1.9$ & $1.2 \pm 1.5$ & 0.010 \\
\hline $\begin{array}{l}\text { Fertilization } \\
\text { rate (\%) }\end{array}$ & $70.4 \pm 28.5$ & $71.3 \pm 34.9$ & 0.900 & $74.6 \pm 29.4$ & $74.6 \pm 35.9$ & 0.997 \\
\hline $\begin{array}{l}\text { No. of Day } \\
3 \text { embryos (n) }\end{array}$ & $2.7 \pm 2.1$ & $1.0 \pm 1.1$ & $\begin{array}{c}< \\
0.001\end{array}$ & $1.8 \pm 1.8$ & $1.0 \pm 1.4$ & 0.007 \\
\hline $\begin{array}{l}\text { No. of top- } \\
\text { quality Day } \\
3 \text { embryos (n) }\end{array}$ & $1.2 \pm 1.5$ & $0.2 \pm 0.4$ & $\begin{array}{c}< \\
0.001\end{array}$ & $0.8 \pm 1.2$ & $0.3 \pm 0.6$ & $\begin{array}{c}< \\
0.001\end{array}$ \\
\hline $\begin{array}{l}\text { Clinical } \\
\text { pregnancy } \\
\text { rate }(\%)\end{array}$ & 22.8 & 5.8 & 0.009 & 16.5 & 8.1 & 0.117 \\
\hline $\begin{array}{l}\text { Live birth } \\
\text { rate } \%(\%)\end{array}$ & 19.0 & 3.8 & 0.012 & 11.3 & 4.8 & 0.152 \\
\hline
\end{tabular}

Data are presented as mean \pm standard deviation and $\%$.

FSH, follicle stimulation hormone 
Figures

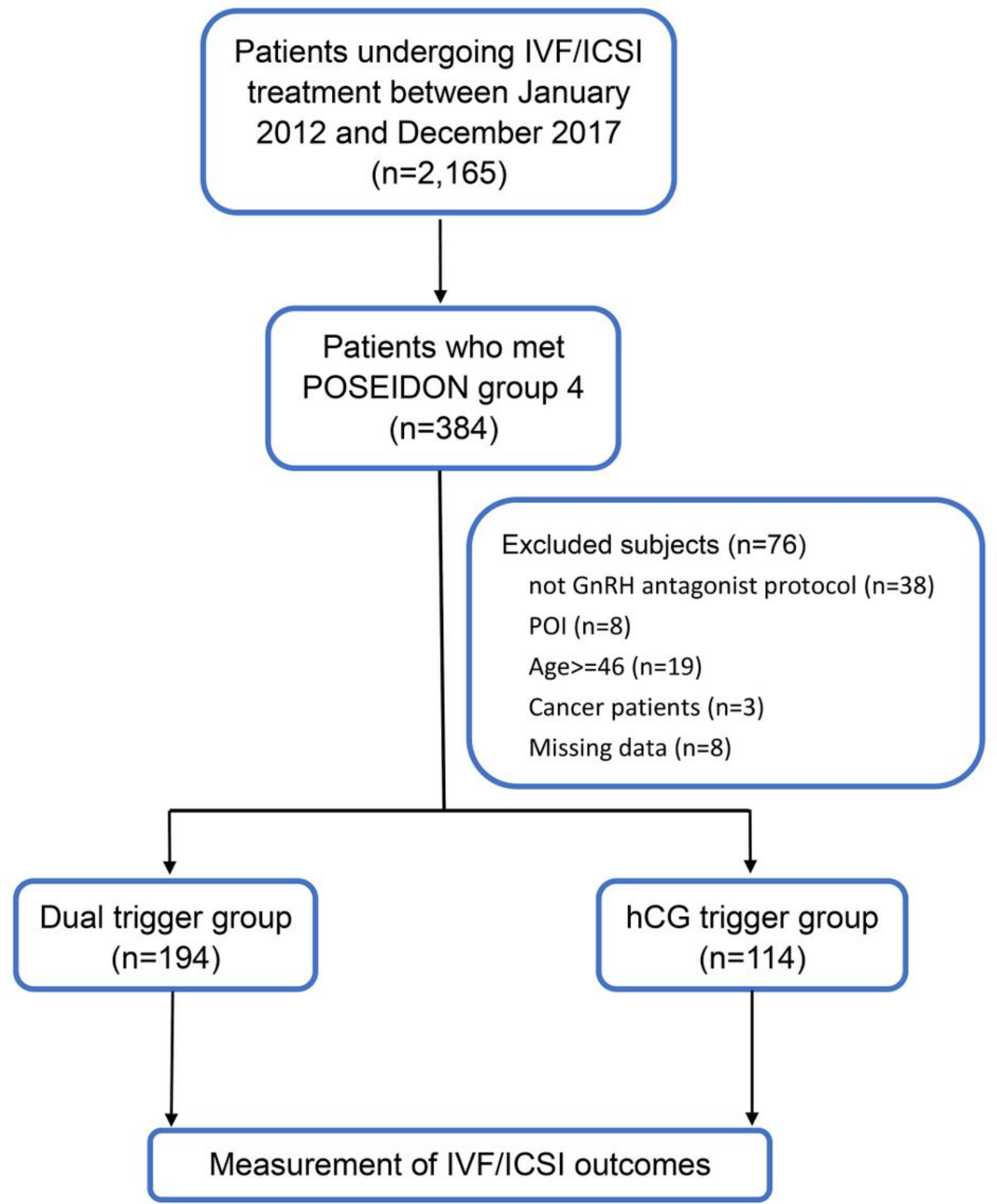

Figure 1

The study flow chart 